\title{
La tradition musicale au Collège Joliette
}

\section{Raymond Locat}

Volume 54, 1987

Culture et religion dans la région de Lanaudière

URI : https://id.erudit.org/iderudit/1006964ar

DOI : https://doi.org/10.7202/1006964ar

Aller au sommaire du numéro

Éditeur(s)

Les Éditions Historia Ecclesiæ Catholicæ Canadensis Inc.

ISSN

0318-6172 (imprimé)

1927-7067 (numérique)

Découvrir la revue

Citer cet article

Locat, R. (1987). La tradition musicale au Collège Joliette. Sessions d'étude Société canadienne d'histoire de l'Église catholique, 54, 83-94.

https://doi.org/10.7202/1006964ar
Résumé de l'article

Cette communication a été donnée devant des congressistes dont plusieursont vécu des événements racontés et dont certains même en ont étéles acteurs. De ce fait, plusieurs épisodes ont été choisis pour ce qu'ils représententparmi leurs souvenirs. Sans changer substantiellement le contenudu récit, le ton aurait été différent si l'exposé avait été destiné à desgens complètement étrangers aux épisodes racontés ou si le temps réservéà la conférence n’avait pas imposé ses limites.Cette réserve nous permet de croire qu'on nous passera aisément certainesallusions un peu hâtives et qui tiennent moins au procédé scientifiquequ'au souci de rendre l'exposé oral le moins terne possible à desauditeurs désireux qu'on fasse le lien avec ce qu'ils connaissent déjà.
Tous droits réservés @ Les Éditions Historia Ecclesiæ Catholicæ Canadensis Inc., 1987
Ce document est protégé par la loi sur le droit d'auteur. L’utilisation des services d’Érudit (y compris la reproduction) est assujettie à sa politique d'utilisation que vous pouvez consulter en ligne.

https://apropos.erudit.org/fr/usagers/politique-dutilisation/ 


\title{
La tradition musicale au Collège Joliette
}

\author{
Raymond LOCAT \\ École polyvalente des Chutes \\ Rawdon, Québec
}

\section{RÉSUMÉ}

Cette communication a été donnée devant des congressistes dont plusieurs ont vécu des événements racontés et dont certains même en ont été les acteurs. De ce fait, plusieurs épisodes ont été choisis pour ce qu'ils représentent parmi leurs souvenirs. Sans changer substantiellement le contenu du récit, le ton aurait été différent si l'exposé avait été destiné à des gens complètement étrangers aux épisodes racontés ou si le temps réservé à la conférence n' avait pas imposé ses limites.

Cette réserve nous permet de croire qu'on nous passera aisément certaines allusions un peu hâtives et qui tiennent moins au procédé scientifique qu'au souci de rendre l'exposé oral le moins terne possible à des auditeurs désireux qu'on fasse le lien avec ce qu'ils connaissent déjà.

Un auditoire d'historiens, avertis ou amateurs, ne veut pas s'entendre répéter ce qu'il sait déjà, comme par exemple l'extraordinaire vitalité des 20 récentes années de musique à Joliette.

Depuis les temps reculés où le grand vicaire Antoine Manseau donnait dans la sacristie de son presbytère de L'Industrie des leçons de chant à des enfants de sa nouvelle paroisse jusqu'à l'époque des réalisations grandioses du Festival de Lanaudière, il y a un long cheminement, une marche progressive à peu près régulière, et c'est ce qu'il nous paraît important de mettre en lumière. Ce qui est aujourd'hui ne peut être en effet le fruit d'une génération spontanée. Il a fallu pas moins d'un siècle et demi d'histoire pour préparer le triomphe que l'on connaît présentement. Des animateurs décidément hors pair ont marqué ponctuellement l'histoire de la musique d'ici, d'autres ont pris la relève, ont donné à ces mouvements un nouveau 
souffle pour les mener aujourd'hui à un épanouissement sans doute exceptionnel dans les annales de la musique à travers le monde.

Contrairement aux tendances générales de l'histoire, dès les début du Collège Joliette, il y a 140 ans, un départ vigoureux et qui sera soutenu était donné par un artiste et pédagogue d'envergure, à propos duquel Gilles Potvin écrit dans le Dictionnaire biographique des musiciens canadiens qu'il «a exercé une heureuse influence sur les débuts en musique d'Ernest et Gustave Gagnon, de Jean-Marie Panneton et qu'il a formé nombre d'autres musiciens de marque». Il s'agit du Frère Louis Vadeboncoeur, c.s.v. Entré au noviciat en 1847, on peut dire que jusqu'en 1893 il ne s'éloignera pas beaucoup de Joliette ni du collège. Pour être exact, disons qu'il fut 26 ans au collège (beaucoup moins que le Père Brunelle).

On croit que dès les débuts du collège on y donnait aussi des leçons de divers instruments, du moins de cuivre, puisque trois ans après, à l'inauguration de la voie ferrée L'Industrie-Lanoraie que $\mathrm{M}^{\mathrm{gr}}$ Bourget vint bénir, la «bande» du collège accompagna les élèves pour se rendre à l'arrivée du premier train. Dès cette année 1850, Alexandre Trottier commence à enseigner le piano et Gaspard Beaudoin continue des leçons de violon. Un frère, Alfred Martel, figurera aussi, dont le neveu, Oscar Martel, sera un des violonistes les plus en vue de Montréal et viendra en 1886 donner un concert au profit de la Chapelle du Sacré-Cœur qu'on ne finissait plus d'embellir.

Et l'histoire se continue, largement dominée par la présence habituelle du Frère Vadeboncoeur, artiste sur tous les points. Dans la liste du personnel, on verra le nom de plusieurs séminaristes. Car il y aura longtemps des études théologiques à l'intérieur du collège. Plusieurs séminaristes seront tour à tour responsables du plain-chant, une façon pour ces étudiants d'être utiles à la maison qui les hébergeait. On cite parmi eux Olivier Dufault. Il paraît que pour Olivier Dufault déjà, à 12 ans, le plain-chant n'avait plus aucun secret. Je ne le dis pas parce que c'est mon grand-oncle, mais parce que Charles Dugas mentionne le fait dans ses Gerbes de souvenir. On note dans la Gazette de Joliette de juillet 1867 que parmi les prix de fin d'année figurent ceux de musique vocale, de piano et de plain-chant.

Saluons l'année 1871 qui vit la fondation de l'harmonie officielle du collège par le Frère Vadeboncoeur. On cessera vraisemblablement de l'appeler la «bande» pour lui donner alternativement divers noms, ccmme fanfare, philharmonie, sans qu'on soit fixé sur une appellation définitive. À son retour d'un voyage de deux ans en Europe, le Frère Vadeboncoeur tenait à ce qu'on l'appelle non pas la fanfare, mais la «fanfare», écrit Charles Dugas, qui continue: «La fanfare du Collège nous précédait, les étudiants, dans nos sorties ou promenades, régularisant nos pas, charmant notre 
marche.» $\mathrm{Il}$ ajoute un trait qui en dit long sur la tradition de distinction qui a toujours caractérisé la population depuis le temps de l'honorable Barthélemy Joliette: «Ces sorties ou «campagnes» dit-il, rappellent le souvenir plus lointain des sorties des élèves où l'on faisait connaissance avec les principaux citoyens et où l'on apprenait d'eux cette belle politesse française qui fait toujours l'ornement de la société joliettaine et lui vient en droite ligne de ses premiers fondateurs et seigneurs. Il me semble voir encore Monsieur de Lanaudière tenir son chapeau bas, tout le temps que défilait devant lui la troupe des écoliers. Comment n'être pas polis et prévenants après de tels exemples de respect et de savoir-vivre?»

Puis vint le Frère Joseph-Octave Maynard, suivi du Père Joseph Charlebois, professeur et responsable de la fanfare avec Anthime Lavigne, un des rares séminaristes qui a continué de faire beaucoup de musique au collège après être devenu prêtre. Antonio Beaudoin s'est fait remarquer comme professeur de piano et il y a enseigné en tout une dizaine d'années. Mentionnons aussi comme professeur le Frère Aristide Fyfe. Parmi les grands noms de cette fin de siècle, on rencontre celui du Frère Étienne Desserres qui, en février 1896, met sur pied le premier orchestre du collège. un nouveau venu apparaîtra à l'aurore de notre siècle, le Frère Philippe Dubé, qui sera d'abord directeur et de la fanfare et de l'orchestre, et qui s'occupera de l'orchestre jusqu'en 1918, tout en enseignant le plus souvent le piano à des élèves.

On est peut-être curieux à ce moment de l'exposé de savoir à quelle occasion les orchestres pouvaient se produire et dans quelle mesure les divers instrumentistes que le collège a connus pouvaient faire montre de leur talent.

Pour nous tous qui n'avons connu que la somptueuse salle académique devenue la salle Rolland-Brunelle, il est difficile d'imaginer dans quelles conditions les concerts pouvaient être donnés. La salle de récréation dans l'aile de briques a sûrement constitué pendant trois quarts de siècle le lieu habituel de toute activité musicale ou thêâtrale du temps. C'est là qu'on rendait les hommages à l'autorité, qu'il s'agisse de Monseigneur Archambault, du Père Cyrille Beaudry, du Père Michel Roberge, etc. C'est là qu'on célébrait les anniversaires importants, les jubilés, que les finissants fêtaient leur Sainte-Catherine, que les Philosophes junior célébrait leur Saint-Thomas par une soirée toute spéciale, mettant justement à profit les talents particuliers des élèves de cette classe comme acteurs ou musiciens. Il y avait également l'occasion des séances traditionnelles qui prirent de l'importance avec les années, la distribution des prix qui a longtemps clôturé une fête annuelle commencée la veille. Depuis toujours, les musiciens ont tenu à célébrer aussi leur patronne, sainte Cécile, par une soirée 
pleine d'improvisation, de découverte et d'émerveillement pour l'ensemble des élèves. Il fallait beaucoup de débrouillardise pour préparer des soirées musicales en s'accommodant de lieux déjà occupés par les mouvements habituels des élèves, et encore plus d'ingéniosité quand il fallait préparer des scènes théâtrales qui devaient dissimuler les surprises jusqu'au dernier moment.

Un personnage qui fera beaucoup parler de lui au début du siècle c'est M. Adélard Ringuet, musicien de grand talent, professeur de piano et de vioion. Îl connaissait bien les vents, jouait du cornet. Quand il vint demeurer à Joliette, à partir de décembre 1901, il arrivait des États-Unis où il avait dirigé plusieurs fanfares. Or, à Joliette, on est en pleine crise à propos de l'harmonie fondée huit ans plus tôt, et la présence de Monsieur Ringuet occasionnera, sans qu'il le veuille, la dissolution de ce corps musical et la fondation de l'Union musicale en février 1902, dont M. Émile Prévost deviendra le chef quatre ans plus tard et pour très longtemps. Monsieur Ringuet y apportera son précieux concours, y fondera un orchestre et ne sera pas absent de la formation que les élèves du collège recevront à cette époque. Il commença en effet à donner des cours de musique tous les soirs à l'Union musicale ainsi qu'au collège. Ce n'est qu'à regret et pour des raisons tout à fait étrangères à la musique que Joliette dut se priver assez tôt des services de l'un des meilleurs musiciens que notre ville a pu connaître.

Avant de poursuivre notre chronologie dans le nouveau siècle, il nous faut analyser l'impact qu'ont pu avoir sur les élèves du collège les concerts donnés par des artistes venus de l'extérieur. Ces concerts ont le plus souvent été donnés, il est vrai, en dehors des murs du collège. La salle de l'institut était en construction quand une artiste internationale, Emma Lajeunesse, surnommée Albani, vint à Joliette le 17 octobre 1857. Charmante enfant de huit ans, elle chantait déjà en cinq langues et lisait les pièces les plus difficiles à première vue, nous dit l'Encyclopédie de la musique au Canada. Il serait intéressant de savoir si nos élèves - ou du moins certains élèves - ont eu l'occasion d'entendre cette artiste prodige. C'est en tous cas le premier concert d'importance, à notre connaissance, dont il soit fait mention à Joliette.

L'exemple le plus ancien d'un genre de soirée dramatique et musicale remonte à 1859 . On célèbre la Saint-Jean-Baptiste dans une soirée au bénéfice de l'institut organisée par une compagnie d'amateurs. Durant l'intermède, indique l'affiche, il sera chanté quelques morceaux choisis d'opéra et un orchestre éxécutera durant la soirée divers morceaux nouveaux. Tel est le contenu musical du programme, toujours vague à souhait, nous laissant chercher de quel orchestre et de quels chanteurs il s'agit. $L a$ Gazette de Joliette de juillet 1866 annonce le concert de Messieurs Oscar 
Martel et Salomon Mazurette, tous deux élèves de Jules Hone et de Monsieur Letondal de Montréal. Dix ans après, ce sera un groupe formé de Calixa Lavallée, Guillaume Couture baryton et le violoniste Jéhin Prume.

C'est ainsi que progressivement les spectacles commencent à prendre de l'importance à Joliette. Un rédacteur un peu emballé écrit même dans la Gazette de Joliette en 1867: «Le temps est aux concerts. À Montréal il en pleut. À Sorel, il vient de s'en faire un des plus gracieux, et à Joliette où l'on raffole de musique, on n'entend que cela, on ne rêve que cela et l'on ne vit que de cela.» En ce qui concerne notre propos, les concerts intra muros et les efforts des musiciens élèves et de leurs professeurs ont eu sans doute une influence plus profonde que ces quelques soirées où des élèves choisis parmi bien d'autres ont pu assister aux performances qui se donnaient à l'extérieur.

Toujours est-il qu'au début du siècle c'était le temps des concerts donnés par des artistes comme les Laurendeau, Clovis et Arthur, chanteurs qui venaient de terminer leurs études classiques, Eugénie Chevalier, soprano, Alphonse et Hector Dansereau, encore jeunes, et plus tard Paul Dufault (encore un Dufault), Albert Chamberland, Joseph Saucier baryton, Annette Lasalle violoniste, Camille Bernard, Maria Thibodeau. Tout cela sans oublier l'influence sur le collège des années glorieuses de l'Union musicale de Joliette. Brillante année 1913 surtout, avec un renfort de musiciens qui acceptaient de venir d'Europe si on leur trouvait un emploi au Canadien national. Une vingtaine de musiciens chevronnés arrivent avec leurs instruments, ce qui permet à l'Union musicale de choisir des pièces très difficiles d'exécution. Impossible qu'une telle présence soit ignorée des jeunes musiciens du collège (devenu depuis 1904 le séminaire). Et quel stimulant pour les jeunes qui ont pu admirer ces musiciens. Malheureusement ces artistes furent rappelés par leur régiment respectif après le début de la guerre de 1914.

Pendant ce temps, à l'intérieur des murs il y a certains élèves dont on n'a pas fini d'entendre parler. Ils possèdent des voix au timbre exceptionnel. Ce sont Lucien Dugas, Édouard Jetté. On ne dira jamais assez comment ces artistes encore jeunes ont pu par leur assiduité au séminaire développer chez les élèves le goût de la beauté, de la nuance, du fini dans l'expression.

La guerre terminée, la vie musicale connaît un essor particulier au séminaire. Notons la venue d'Eugène Chartier, professeur de violon, alors que le Frère Henri Hurtubise prend la direction de l'orchestre. Eugène Chartier était alors directeur de la Philharmonique de Montréal et de la fameuse Harmonie d'Hochelaga. Notons aussi la naissance de l'orchestre de 
la famille Asselin, formé d'Octavien, également professeur, de sa sœur Alma, pianiste, et des autres membres de la famille: Armand et Cécile violonistes, Julien violoncelliste. Cette famille sera de toutes les soirées artistiques pendant une quinzaine d'années. La présence permanente de cet orchestre dans Joliette aura contribué largement à la diffusion d'une belle musique exécutée avec le raffinement d'artistes accomplis. On mentionne aussi parmi les élèves la présence de Maurice Ducharme, excellent violoniste, selon le Père Brunelle. Le docteur Dionne ajoute qu'il était le meilleur violoniste de Joliette.

De nouveaux chanteurs joliettains commencent aussi à figurer. La Société des amateurs qui existe depuis plusieurs années fait ainsi connaître des artistes locaux comme les Gravel, les Wodon, Masse, Alarie, Paquette, Denis, Désormeaux, etc., qui assurent la continuité entre deux concerts d'artistes montréalais, tels que Charles Marchand avec le pianiste Emest Patience, Lucien Chevalier, Ulysse Paquin, Georges Trépanier, Arthur Laurendeau et combien d'autres.

L'année 1926 allait marquer un tournant dans l'histoire musicale de Joliette et fixer désormais l'attention sur la célèbre salle académique dont la construction venait de se terminer. Événement dont l'impact est comparable à celui qu'aura sans doute l'ouverture en 1989 du déjà célèbre amphithéâtre et salle de concert si ardemment souhaité pour Joliette. Imaginez en 1926 ce déplacement soudain d'intérêt vers la merveille du temps, qui fait pâlir d'envie les voisins et qui deviendra pour les élèves du Collège un lieu accessible, un lieu enfin autorisé pour entendre des artistes de l'extérieur que le règlement et l'encadrement les empêchaient jusque-là d'approcher. Dès le mois de juin c'est déjà un triomphe avec la venue de 70 musiciens de la Symphonie du Conservatoire national affilié à l'Université de Montréal sous la direction du professeur Eugène Chartier déjà bien connu des joliettains.

On aimerait connaître les promoteurs de cette salle de concert et de spectacle qui devait occuper un si large espace à l'intérieur de l'aile Bonin. Étienne Marion (rhétorique), pianiste, avait dû trouver un peu vulgaire de s'exécuter dans une salle de récréation; Lucien Bellemare (versification) voulait sans doute plus de place pour sa contrebasse. Et qui sait? Rolland Brunelle, Émile Jetté, Paul Duclos et même Paul Dionne ont peut être posé cette condition de la construction d'une salle pour décider de leur entrée imminente au collège... Toujours est-il que cette nouvelle salle va soulever un enthousiasme dont les répercussions seraient longues à analyser. Quelle joie d'entendre enfin et de voir nos artistes joliettains encore assez peu connus des élèves! Lucien Dugas, qui vient de fonder l'Association des chanteurs avec Jean-Paul Courteau, fera appel à Jean Riddez de l'Opéra de 
Paris qui viendra donner des cours de chant. Les artistes joliettains ou étrangers préféreront, on imagine bien, cette salle opulente à la salle du marché ou au Théâtre Passe-temps où, jusque-là, les grandes vedettes s'étaient fait entendre. À l'intérieur même des murs, deux élèves de philosophie, Edmond Bellemare, violoniste, et Roger Deshaies, clarinettiste, pouvaient déjà en donner plein les oreilles à leurs camarades au concert de la Sainte-Cécile.

Un dynamisme sans retour animera désormais le séminaire sur le plan musical. Des musiciens comme ceux que l'on a mentionnés un peu plus haut vont comme par hasard se trouver en même temps au collège, et leur talent de solistes ne tardera pas à se manifester. C'est le cas en particulier du jeune Rolland Brunelle déjà connu au collège avant même d'y entrer puisque le Père Jean Perreault l'avait déjà amené quand il avait dix ans et lui avait fait jouer du violon devant les autres musiciens de la fanfare. Ce qui est tout à fait exceptionnel dans l'histoire de la musique chez les Clercs de Saint-Viateur c'est que Rolland Brunelle commençait une carrière de soliste et, plus tard, d'éducateur musical qui allait battre bien des records. Quand, à la Sainte-Cécile de 1927, il jouait Scène de ballet de C. de Bériot, pendant que, le même soir, Étienne Marion finissant jouait un concert polonais de J.H. Haydn, il était bien loin de penser que, exactement 60 ans plus tard, cette même salle académique porterait son nom et qu'il aurait devant lui son orchestre $\mathrm{A}$, son orchestre $\mathrm{B}$, et des dizaines de petits violons qu'il dirigerait avec le même dynamisme et d'une main aussi alerte que lorsqu'il exécutait son premier solo de jeune collégien. Ce n'est un secret pour personne qu'en 1989 ce sera le $50^{e}$ anniversaire d'enseignement de la musique du Père Brunelle au Séminaire de Joliette.

Il faut rendre justice ici à tous les membres de la famille Lomer Brunelle qui ont marqué, par la qualité de leur participation, l'histoire de la musique, à Joliette d'abord, dans un orchestre familial, puis dans les répétitions et concerts au séminaire. Nommons en particulier Jean-Paul, flutiste, Maurice hauboiste, Gérald, clarinettiste, Rolland, violoniste. Leur présence a permis pendant plusieurs années de maintenir la solidité de l'orchestre et assurer la continuité nécessaire au sein du groupe des étudiants musiciens dont le passage à l'orchestre était forcément temporaire.

Dès l'année 1929, Rolland Brunelle formera avec quelques autres chanteurs les Troubadours de L'Industrie qui propageront le folklore et chanteront des chants canadiens harmonisés. À l'occasion des vacances, Rolland Brunelle, étudiant, se joindra à l'Union musicale de Joliette y jouant tour à tour tous les cuivres. Quand il sera en Philosophie junior, l'harmonie de sa classe jouera trois pièces de sa composition. Pour tout dire, il en sera de Rolland Brunelle comme du Frère Vadeboncoeur. Partout 
où il se trouvera, les musiciens auront envie de se grouper et de faire de la musique. On verra même, quelques années après, son groupe du scolasticat d'études théologiques venir offrir un concert au séminaire - fait inouï - et le Frère Brunelle y jouera le Concerto en do de Haydn.

Un autre élève dont devraient être fiers les abbés Alphonse Fafard et Léo Paradis, alors directeurs respectifs de l'harmonie et de l'orchestre, c'est Paul Dionne, qui en Philosophie junior, en 1933 joua le très difficile concerto de Boccalari à la clarinette. Celui que l'on connaît sous le nom de dúctéur Dionne a été un des piliers de la musique au séminaire comme élève puis comme ancien élève, attaché à son Alma Mater où il passait la plus grande partie de ses loisirs en se joignant à l'orchestre et y apportant son précieux concours. Nommé par l'abbé Fafard à l'automne de 1928 premier soliste des clarinettes alors qu'il n'était qu'en syntaxe, il a conservé ce poste pendant plus de 30 ans. Le docteur Dionne fut sans aucun doute le professionnel joliettain qui participa le plus longtemps aux activités de l'orchestre. Il a personnifié en quelque sorte la permanence de la tradition musicale du séminaire pendant plus d'un demi-siècle. Encore ici, c'est toutc la famille Dionne, cinq autres musiciens et musiciennes, qu'il faudrait nommer pour leur contribution assidue à l'orchestre.

Dès 1935, le Père Étienne Marion commencera une carrière de professeur, de lettres surtout, et joindra à cette tâche plusieurs activités musicales discrètes mais de grande qualité. Il commencera une série de cours d'initiation musicale, fondera un cercle musical avec audition et exécution de chant et sera le pianiste attitré et le critique musical et littéraire dont on craindra les foudres. Il sera aussi un organiste un peu particulier, sacrifiant parfois la rigueur et les détails techniques aux impératifs de la création. À ce titre, il demeure un des grands improvisateurs, sans doute le plus grand qui ait touché les grandes orgues du séminaire, paraphrasant avec une facilité étonnante, au moment des vêpres du dimanche par exemple, les thèmes de l'opéra ou de la symphonie entendus à la radio durant l'après-midi. Au hasard des soirées données au séminaire, on lit: «Ce 11 février 1951, à l'heure musicale, réalisation du Père Marion, on entendra de la musique du XVIII ${ }^{e}$ siècle jouée et chantée par des artistes locaux.» Le Père sera aussi responsable des concerts sacrés du Noviciat de Joliette vers les années 1952-1959, un avant-goût des concerts Pro organo que nous connaissons.

À cette époque, le Père Wilfrid Corbeil était déjà connu des étudiants. Professeur de lettres, la majorité des élèves le connaissaient surtout pour les décors somptueux qu'il réalisait à l'occasion des grandes pièces de théâtre ou séances de fin d'année. $\Pi$ s'occupa aussi de musique, faisait fonction d'organiste à la chapelle pendant une dizaine d'années. Il fut notamment à l'origine de la fondation des Amis du Séminaire qui permit la 
venue d'artistes de l'étranger. À l'occasion de l'une des saisons artistiques de la Société, le Père tient des propos que Fernand Lindsay ne désavoueraient pas, encore aujourd'hui. Il écrit dans l'un des dépliants de la saison: «La valeur du programme des soirées que la Société des Amis du Séminaire présente cette année est l'heureux aboutissement des efforts qui ont été faits pour donner à Joliette une saison de concerts et de théâtre digne des grandes cités. Nous comptons trouver chez le public de Joliette et des environs l'accueil le plus favorable et nous avons augmenté le prix du billet afin de présenter au public des artistes de qualité supérieure.» Cela s'écrivait il y a au-delà de 40 ans.

À partir des années 1940 donc, une sorte de concertation de forces nouvelles au niveau du personnel favorise en tous points l'essor de la musique au séminaire. Avec la présence et l'action des pères Corbeil, Brunelle, Bellemare, Marion, Jetté, tout devient possible. Pendant une dizaine d'années, c'est le Père Bellemare qui a la direction de l'orchestre comme aussi celle de la chorale.

Fondateur en 1943, avec le Père Brunelle, d'un orchestre symphonique, le Père Bellemare le dirigera avec autorité. Beaucoup d'intuition, une disposition naturelle pour la direction, un sens inné des relations humaines, une audace pour relever des défis comme celui d'obtenir que Wilfrid Pelletier vienne diriger l'orchestre d'ici dans la Symphonie inachevée de Schubert, ou encore le défi de se mesurer à des pièces d'envergure jamais exécutées auparavant, comme des symphonies de Beethoven, des concertos de Mozart, de Mendelssohn, de Grieg, et autres pièces qui mirent en valeur les talents des violonistes Edmond Bellemare, Rolland Brunelle, du clarinettiste Paul Dionne, de pianistes comme Paul Doyon, Georges Lindsay, professeur d'orgue et de piano. Ce dernier, fut l'un de ceux qui ont fait briller d'un vif éclat la période précédant et entourant les fêtes du centenaire du collège. Le Père Bellemare a su également utiliser les connaissances d'écriture musicale de maitre Robert Tellier dont le dévouement à l'orchestre a été total. Enfin le Père s'est acquis le soutien d'une dizaine de musiciens de la Symphonie de Montréal.

Le Pageant du centenaire fut sans nul doute un sommet comme événement artistique et musical, comme l'avait été à sa façon la célèbre réalisation de Jonathas en 1935, dont la direction orchestrale avait été confiée à Eugène Chartier, ce pageant donc était l'aboutissement d'efforts conjugués de plusieurs éléments dynamiques de la population joliettaine. L'orchestre, enrichi de divers artisans de la région et de Montréal, une chorale mixte de 200 voix, un dispositif reliant les grandes orgues du séminaire au théâtre du pageant dressé au fond de la cour des élèves, une chorégraphie de Morenoff, enfin le théâtre en plein air, conception du Père Wilfrid Cor- 
beil. C'est même avec un peu de nostalgie qu'à l'automne de cette même année la chorale du centenaire prolongeait le mythe du pageant en reprenant quelques-uns des grands airs qu'on y avait chantés: l'Ode à Barthélemy Joliette du Père Brunelle, le Vivat de Gabriel Cusson, le Te Deum de Haendel...

La formule des récitals d'élèves commence à prendre de l'ampleur vers ces années-là. Déjà en 1944, on lit le Récital des élèves de Rolland Brunelle, Georges Lindsay et Lucien Dugas, et quelques années après, de Marcel Savaria, prôfesseur de piano. En 1950, on présente une Revue musicale sous la direction du Père Brunelle avec la participation cette fois de plus jeunes élèves qui ne sont pas tous du séminaire. Dans le même temps, étaient fondées les Voix du Printemps, prélude de ce qui deviendra deux ans après les Chanteurs de la place Bourget avec René Martin. Dans l'intervalle, les Jeunesses musicales sont fondées. Ces nouveaux organismes, joints à l'association des Amis du Séminaire, allaient assurer des saisons artistiques de plus en plus nourries et variées. Impossible ici de détailler, mais peut-on raisonnablement taire les noms de Pierrette Alarie et de Léopold Simoneau, de Gérard Souzay, de Malkuzinski, de Carmen Torrès, de John Newmark, d'Erna Sack, de Paul Tortellière, de Tito Schipa et de combien d'autres?

Il n'échappe à personne que le théâtre avait désormais vécu ses heures de gloire. Les modifications importantes au niveau de l'enseignement, le nombre de plus en plus réduit de pensionnaires de nos collèges, des horaires radicalement changés et combien d'autres causes portèrent un dur coup aux productions artisanales. Curieusement, cette évolution n'allait pas affecter le côté musical, ou plutôt allait le forcer à une orientation différente, à une transformation axée sur l'accessibilité de tous les talents à la formation musicale classique. Lors du concert de la Sainte-Cécile en 1958, on trouvait à la fin du programme un étrange questionnaire, et ce n'était pas le premier du genre: «Pouvez-vous diviser les instruments de l'orchestre? Quels instruments ce soir ont le mieux joué? Énumérez les bienfaits de la musique. Élèves d'éléments, vous avez entendu votre orchestre pour la première fois et vous êtes membres d'un jury, etc.». Ce sont de telles interrogations qui ont remué enfants et parents et soulevé une vague de fond dont les effets se sont fait sentir jusqu'à aujourd'hui.

Au récital des gagnants de 1968, figurent un grand nombre d'élèves au violon, dont Angèle Dubeau, cinq ans, alors que le Père Brunelle a déjà ajouté à sa tâche une assistance à l'Union musicale de Joliette qui montre de l'essoufflement et qu'on maintiendra difficilement jusqu'à sa dissolution en 1980. On peut parler d'une action du Père Brunelle à la base, action constante, incitative tout au long de cette métamorphose. Travailleur opi- 
niâtre, il a mobilisé une pléiade de futurs artistes, soulevé l'enthousiasme de familles entières pour l'étude de la musique classique et contribué pour une part irremplaçable à l'excellence de ce qui existe aujourd'hui.

Il fut admirablement secondé par celui dont on a volontairement retenu le nom jusqu'à présent, peut-être pour couronner plus magnifiquement cet exposé. Dans le temps où Georges Lindsay donnait des cours de piano, il avait invité ses neveux de Trois-Pistoles, Jean-Marc et Fernand, à s'inscrire au Séminaire de Joliette, faisant miroiter devant eux les attraits d'une maison pleine de musique: orchestre, harmonie, chorale. On n'ose pas penser de qui et de quoi nous serions privés si ces élèves avaient choisi un autre collège! Effectivement, ils ont été des élèves très actifs. Fernand en particulier y apprit divers instruments, devint peu à peu le pianiste du collège, fut sans doute l'élève le plus proche des préoccupations du pageant de 1947. Marchant d'un pas rapide, c'était l'élève omniprésent, disponible, s'amusant follement autant avec les matières académiques qu'avec les choses de l'art et du rêve. Mêlé assez tôt à tout ce qui est organisation, quoi de plus facile pour lui que d'en multiplier les formes.

Comme nous le disions au début il serait superflu d'entrer ici dans les détails de ce qui s'impose à l'œil et à l'oreille depuis quelques années. Une édition spéciale du $1^{\text {er }}$ juillet 1987 du Joliette-Journal a été consacrée exclusivement à l'action du Père Lindsay dans la région. Une série de réalisations étonnantes, couronnées en quelque sorte par le Festival d'été qui a déjà acquis une reconnaissance internationale.

On peut lire au dos d'un dépliant des années dernières: «L'enseignement de la musique est à la base de cette réussite régionale. L'Orchestre symphonique des Jeunes de Joliette, les Jeunesses musicales de Joliette, sous la responsabilité du Père Lindsay, qui en 1964 se méritaient le trophée national sur 93 autres villes et s'ouvraient une voie vers le Camp musical d'Orford, la création par le Père Lindsay du festival-concours en 1962, du Camp musical de Lanaudière en 1967, de l'École de musique du Centre culturel en 1976, et cela joint à la direction depuis 1963 de la chorale mixte des Chanteurs de la place Bourget sont autant d'apports pour l'éducation et l'animation musicale de toute cette région.» Suivent des remerciements à différents organismes publics ou privés qui montrent que des relations de bonne entente avaient été créées depuis longtemps par celui que l'on peut appeler l'homme des relations publiques tout autant que l'animateur de la musique classique d'ici depuis plus de 25 ans et dont l'action soutenue a permis que Joliette devienne un centre musical unique qui figure avec fierté parmi les capitales de la musique. Je me doutais bien que j'allais finir sur le ton du panégyrique. Ce n'est cependant pas le point le plus faible de l'exposé. On pense plutôt aux innombrables lacunes d'un trop rapide sur- 
vol de l'histoire musicale d'un gros collège. Nous espérons combler ce vide d'ici quelques années par un ouvrage qui dépouillera de façon plus exhaustive les trésors de l'histoire musicale - cette fois - et du collège et de Joliette.

D'ores et déjà, on peut conclure qu'avec ses modestes cours de chant donnés à L'Industrie et les prémices d'une formation musicale dont il a eu l'inspiration, Antoine Manseau a inauguré une tradition musicale qui allait se maintenir jusqu'au jour de l'Académie qui, par une de ces rencontres imprévisiules de l'histoire, porte aujourd'hui son nom.

\section{Bibliographie}

A. Sources manuscrites et sonores:

Boîtes ou chemises de Programmes-souvenir conservés aux Archives de la Société historique de Joliette et à la Maison provinciale des Clercs de Saint-Viateur.

Cahier manuscrit du Père Charlebois, liste du personnel, Collège Joliette, 1847-1915.

Enregistrement sonore sur cassette de souvenirs personnels par le Père Brunelle et le docteur Paul Dionne.

\section{B. Sources imprimées:}

Album du centenaire de Joliette, 1864-1964, Edmond Gervais, 1964.

Anonyme (J. Charlebois), Les Anciens du Séminaire: écrivains et artistes, 1927.

Annuaire de l'Institut des Clercs de Saint-Viateur.

Bernard, Antoine, Les Clercs de Saint-Viateur au Canada, tome 1, 1947.

Dugas, Charles, Gerbes de Souvenir, 2 vol., Montréal, Arbour et Dupont, 1914.

La Gazette de Joliette.

Potvin, Gilles, Dictionnaire biographique des musiciens canadiens.

L'Union musicale, 1901-1946, brochure publiée par l'Action populaire de Joliette, 1946. 